Paper

\title{
An analysis of delay-locked loop tracking binary Markovian sequence in the presence of multiple access interference and channel noise
}

\author{
Keisuke Nagata ${ }^{1 a)}$, Hisato Fujisaka ${ }^{1}$, Takeshi Kamio ${ }^{1}$, \\ and Kazuhisa Haeiwa ${ }^{1}$ \\ ${ }^{1}$ Hiroshima City University \\ 3-4-1 Ozuka-higashi, Asa-minami-ku, Hiroshima 731-3194, Japan \\ a) keisuke@sp.info.hiroshima-cu.ac.jp
}

Received February 1, 2011; Revised May 13, 2011; Published October 1, 2011

\begin{abstract}
This paper presents an analysis of delay-locked loops in direct spreading code division multiple access systems with Markovian spreading sequences. The established analysis with piecewise constant approximation is similar to the mean field analysis which estimates macroscopic characteristics of a spatially-distributed probabilistic system from the local interaction between the elements of the system with low computational complexity. The low complexity makes it possible to analyze the behavior of not only second-order archetype delaylocked loops but also higher-order non-coherent delay-locked loops in the presence of both multiple access interference and channel noise. It is found from the presented analysis that replacing i.i.d. spreading sequences with Markovian spreading sequences with non-vanishing negative autocorrelation reduces the phase tracking error of a non-coherent $1 \Delta$ delay-locked loop. The time spent for estimating the error by the presented method is about 1/1000 compared with that by the path integral method.
\end{abstract}

Key Words: code division multiple access, delay-locked loop, Markovian sequence, mean field analysis, stochastic difference equation, piecewise constant function

\section{Introduction}

In direct spreading code division multiple access (DS-CDMA) communications, delay-locked loops (DLL) are often used for locally generated sequences to synchronize with received spread-spectrum signals in despreading process at receivers $[1,2]$. Since the synchronization is one of nonlinear phenomena, the rigorous analysis of DLLs is difficult. In most of the preceding DLL analyses [1-5], small synchronization error allows DLLs to be linearized. Widely utilized spreading sequences such as Gold codes are regarded as independent and identically distributed (i.i.d.) sequences. Then, in the analyses, multiple access interferences (MAI) are approximated by white Gaussian noise (WGN). 
In recent years, it has been reported that Markovian spreading sequences with non-vanishing negative autocorrelation decrease MAI in asynchronous DS-CDMA communications [6-13]. When Markovian sequences are employed in DS-CDMA, MAI is not considered as white noise any more. Thus, a novel estimation technique of the synchronization error of DLLs must be developed.

Recently, it is reported that the preceding analyses can not evaluate the dependency of the synchronization error on the statistical characteristics of spreading sequences and that a kind of path integral estimates the error with high precision [14]. However, because of high computational complexity of the path integral method, the DLLs to be analyzed are restricted to archetype DLLs which suffer from MAI but are free from communication channel noise.

The mean field analysis (MFA) [15-17] estimates macroscopic characteristics of a spatiallydistributed probabilistic system from the local interaction between the elements of the system with low computational complexity. The MFA is actively applied in recent years not only to statistical mechanical systems but also to information processing and communication systems [16-18]. For example, the MFA is used for detecting received signals in CDMA communications by exploiting an analogy between CDMA systems and statistical mechanical systems consisting of large elements [18]. Although a DLL is not a spatially-distributed system at all, we find that its probabilistic behavior is described similarly to an evolution of an all-coupled spatially-distributed probabilistic system. Then, in this paper, we will attempt to apply a similar method to the MFA to estimate the synchronization error of higher-order non-coherent DLLs in the presence of both MAI and channel noise.

The rest of this paper is organized as follows: In Section 2, a non-coherent $1 \Delta$-DLL is introduced and described with common and differential mode components of its correlator outputs. In Section 3 , it is shown that the evolution of the probability distribution of the state of the DLL is represented like the evolution of that of a spatially-distributed infinite-range probabilistic system by quantizing channel noise. In Section 4, an MFA-like procedure can be applied to the computation of the moments of the DLL state by using piecewise constant approximation. In Section 5, results of the computation of the moments and their dependency on the eigenvalue of Markovian spreading sequences are shown. Section 6 concludes this paper.

\section{Delay-locked loop}

\subsection{Non-coherent $1 \Delta$-DLL under MAI and channel noise}

In the despreading process at receivers of DS-CDMA systems, DLLs synchronize their local sequence with one of received spread-spectrum signals. The synchronization may be called phase tracking. Let the number of CDMA users be $J_{I}+1, J_{I}(\geq 1)$ : integer. We denote the spreading code signal and the data signal of the $i$-th user by $c^{(i)}(t)$ and $d^{(i)}(t)$ respectively and define them as follows:

$$
\begin{aligned}
& c^{(i)}(t)=\sum_{n=-\infty}^{+\infty} c_{n}^{(i)} \operatorname{rect}_{\Delta}(t-\Delta n), \quad c_{n}^{(i)} \in\{+1,-1\}, \\
& d^{(i)}(t)=\sum_{m=-\infty}^{+\infty} d_{m}^{(i)} \operatorname{rect}_{L \Delta}(t-L \Delta m), \quad d_{m}^{(i)} \in\{+1,-1\}, \\
& \operatorname{rect}_{T}(t)= \begin{cases}1 & \text { for } 0 \leq t \leq T \\
0 & \text { otherwise }\end{cases} \\
& i \in\left\{1,2, \cdots, J_{I}+1\right\}
\end{aligned}
$$

where $\Delta$ and $L \Delta, L(\gg 1)$ : integer, denote the durations of one-chip of spreading code signals and one-bit of data signals. Each DLL receives the sum of spread-spectrum signals $d^{(i)} c^{(i)}$ with different time delays $t_{I}^{(i)}$ and an additive common channel noise $\Gamma(t)$,

$$
\sum_{i=1}^{J_{I}+1} d^{(i)}\left(t-t_{I}^{(i)}\right) c^{(i)}\left(t-t_{I}^{(i)}\right)+\Gamma(t)
$$

Let the delay difference be expressed with $\Delta$ as $t_{I}^{(i)}-t_{I}^{(j)}=h_{i, j} \Delta$. In this paper, DLLs are considered to be used in asynchronous DS-CDMA communications. Therefore, $h_{i, j} \in[0,1]$ for any $i$ and $j \in$ 


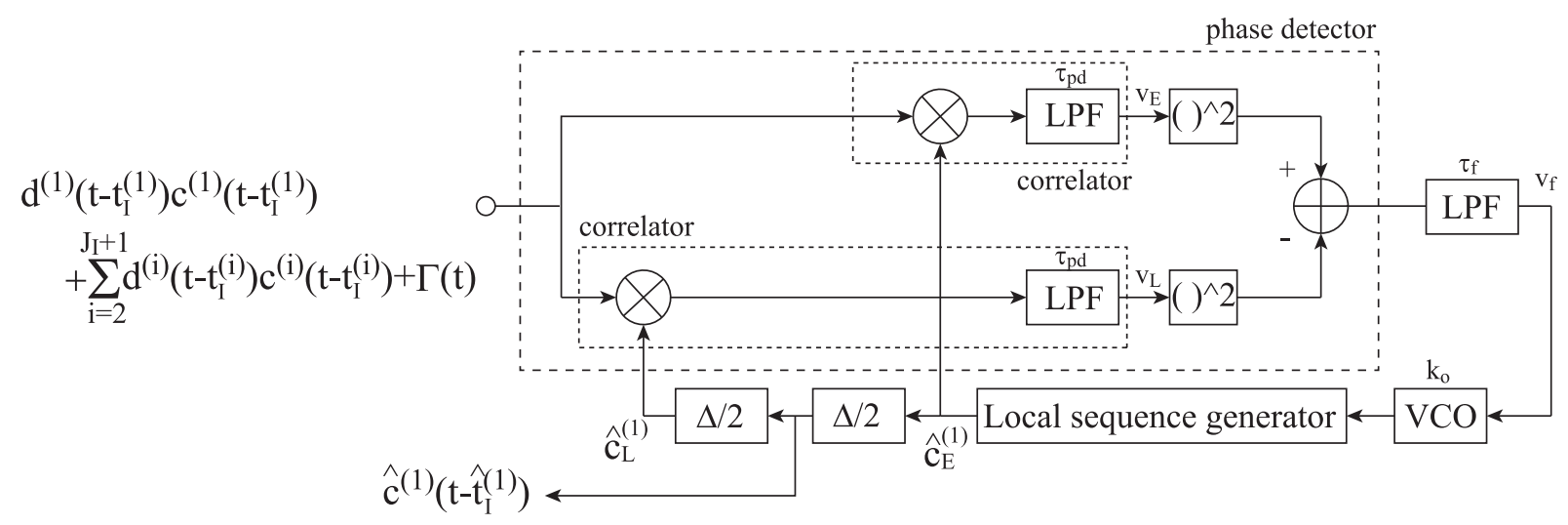

Fig. 1. Non-coherent $1 \Delta$ delay-locked loop.

$\left\{1,2, \cdots, J_{I}+1\right\}$. The spreading sequences $c^{(i)}$ are Markovian sequences changing their binary values at probability $S$. Their chip transition is described by the following transition matrix with $S$ or its eigenvalue $\lambda$ :

$$
\left(\begin{array}{cc}
1-S & S \\
S & 1-S
\end{array}\right)=\frac{1}{2}\left(\begin{array}{cc}
1+\lambda & 1-\lambda \\
1-\lambda & 1+\lambda
\end{array}\right), \quad 0 \leq S \leq 1, \quad-1 \leq \lambda \leq 1
$$

A non-coherent $1 \Delta$-DLL consists of a phase detector (PD), a lowpass loop filter (LPF), and a local sequence generator driven by a voltage controlled oscillator (VCO), as shown in Fig. 1. The PD is built of two correlators, two squarers, and an adder. Assume that a DLL synchronizes its local sequence $\hat{c}^{(1)}\left(t-\hat{t}_{I}^{(1)}\right) \in\{+1,-1\}$ with one of the spread-spectrum data signal $d^{(1)} c^{(1)}$. For the DLL, MAI signal is given by

$$
\text { MAI : } \sum_{i=2}^{J_{I}+1} d^{(i)}\left(t-t_{I}^{(i)}\right) c^{(i)}\left(t-t_{I}^{(i)}\right)
$$

The local sequence generator supplies the following early and late sequences $\hat{c}_{E}^{(1)}, \hat{c}_{L}^{(1)}$ to PD:

$$
\hat{c}_{E}^{(1)}\left(t-\hat{t}_{I}^{(1)}\right) \equiv \hat{c}^{(1)}\left(t-\hat{t}_{I}^{(1)}+\frac{1}{2} \Delta\right), \quad \hat{c}_{L}^{(1)}\left(t-\hat{t}_{I}^{(1)}\right) \equiv \hat{c}^{(1)}\left(t-\hat{t}_{I}^{(1)}-\frac{1}{2} \Delta\right)
$$

The phase tracking error of the DLL is defined by

$$
\varepsilon(t) \equiv t_{I}^{(1)}-\hat{t}_{I}^{(1)}
$$

The correlation between $c^{(1)}$ and $\hat{c}^{(1)}$

$$
R(\varepsilon) \equiv \frac{1}{L \Delta} \int_{t_{0}}^{L \Delta+t_{0}} c^{(1)}\left(t-t_{I}^{(1)}\right) \hat{c}^{(1)}\left(t-t_{I}^{(1)}+\varepsilon\right) d t, \quad t_{o}: \text { any time }
$$

is characterized as shown in Fig. 2(a). The product of the MAI signal and $\hat{c}^{(1)}$ and the product of $\Gamma$ and $\hat{c}^{(1)}$ are zero in average. Then, when the time constant $\tau_{p d}$ of the two lowpass filters in the correlators is $\Delta<\tau_{p d}<L \Delta$, the outputs of the two filters are approximated by

$$
\begin{aligned}
v_{E}(t) & \approx d^{(1)}\left(t-t_{I}^{(1)}\right) R\left(\varepsilon+\frac{\Delta}{2}\right) \\
v_{L}(t) & \approx d^{(1)}\left(t-t_{I}^{(1)}\right) R\left(\varepsilon-\frac{\Delta}{2}\right) \\
v_{E}^{2}(t)-v_{L}^{2}(t) & \approx R\left(\varepsilon+\frac{\Delta}{2}\right)^{2}-R\left(\varepsilon-\frac{\Delta}{2}\right)^{2}
\end{aligned}
$$

The last equation indicates that PD output is almost independent of data signal $d^{(1)}$. Since $\Delta<\tau_{p d}$, we may neglect the difference between $c^{(1)}\left(t-t_{I}^{(1)}\right) \hat{c}_{E / L}^{(1)}\left(t-\hat{t}_{I}^{(1)}\right)$ and $R\left(\varepsilon \pm \frac{\Delta}{2}\right)$. Then, we describe the DLL as follows: 


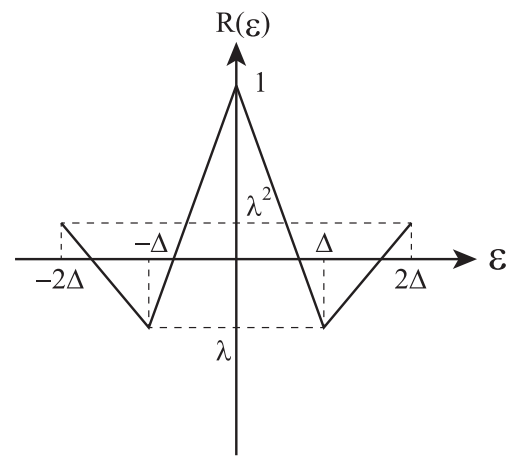

(a) Autocorrelation.

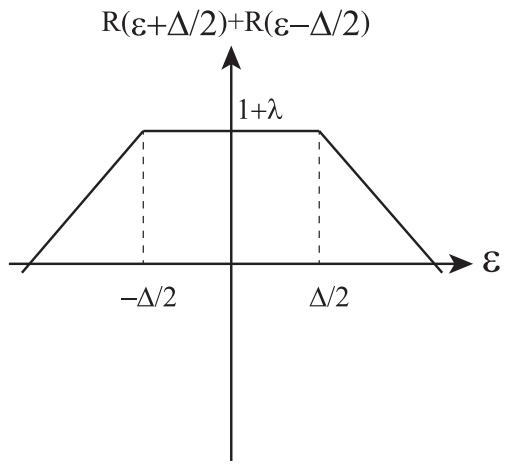

(b) Common component.

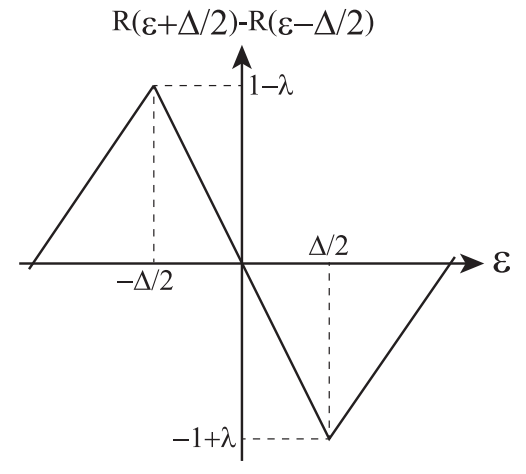

(c) Differential component.

Fig. 2. Correlation characteristic of Markovian sequences and the common and differential components of the phase detector outputs.

$$
\begin{aligned}
\frac{d \varepsilon}{d t} & =k_{o} v_{f} \\
\frac{d v_{E}}{d t} & =\frac{1}{\tau_{p d}}\left(d^{(1)} R\left(\varepsilon+\frac{\Delta}{2}\right)+\hat{c}_{E}^{(1)} \sum_{i=2}^{J_{I}+1} d^{(i)} c^{(i)}+\hat{c}_{E}^{(1)} \Gamma-v_{E}\right) \\
\frac{d v_{L}}{d t} & =\frac{1}{\tau_{p d}}\left(d^{(1)} R\left(\varepsilon-\frac{\Delta}{2}\right)+\hat{c}_{L}^{(1)} \sum_{i=2}^{J_{I}+1} d^{(i)} c^{(i)}+\hat{c}_{L}^{(1)} \Gamma-v_{L}\right) \\
\frac{d v_{f}}{d t} & =\frac{1}{\tau_{f}}\left(v_{E}^{2}-v_{L}^{2}-v_{f}\right)
\end{aligned}
$$

where $k_{o}$ is the gain of $\mathrm{VCO}, \tau_{p d}$ is the time constant of the lowpass filters in the correlators, and $\tau_{f}$ is the time constant of LPF.

\subsection{Common and differential mode description of phase detection}

We define common and differential mode components of the correlator outputs as

$$
v_{c}=v_{E}+v_{L}, \quad v_{d}=v_{E}-v_{L}
$$

Applying variable transformation (10) to Eq. (9), we obtain

$$
\begin{aligned}
\frac{d \varepsilon}{d t} & =k_{o} v_{f} \\
\frac{d v_{c}}{d t} & =\frac{1}{\tau_{p d}}\left(R\left(\varepsilon+\frac{\Delta}{2}\right)+R\left(\varepsilon-\frac{\Delta}{2}\right)+\left(\hat{c}_{E}^{(1)}+\hat{c}_{L}^{(1)}\right) \sum_{i=2}^{J_{I}+1} d^{(i)} c^{(i)}+\left(\hat{c}_{E}^{(1)}+\hat{c}_{L}^{(1)}\right) \Gamma-v_{c}\right) \\
\frac{d v_{d}}{d t} & =\frac{1}{\tau_{p d}}\left(R\left(\varepsilon+\frac{\Delta}{2}\right)-R\left(\varepsilon-\frac{\Delta}{2}\right)+\left(\hat{c}_{E}^{(1)}-\hat{c}_{L}^{(1)}\right) \sum_{i=2}^{J_{I}+1} d^{(i)} c^{(i)}+\left(\hat{c}_{E}^{(1)}-\hat{c}_{L}^{(1)}\right) \Gamma-v_{d}\right) \\
\frac{d v_{f}}{d t} & =\frac{1}{\tau_{f}}\left(v_{c} v_{d}-v_{f}\right)
\end{aligned}
$$

With the transformation, we removed $d^{(1)}$ since $\tau_{p d}<L \Delta$ and term $v_{c} v_{d}=v_{E}^{2}-v_{L}^{2}$ is independent of $d^{(1)}$. The common and the differential components of the correlation functions, $R\left(\varepsilon+\frac{\Delta}{2}\right)+R\left(\varepsilon-\frac{\Delta}{2}\right)$ 
and $R\left(\varepsilon+\frac{\Delta}{2}\right)-R\left(\varepsilon-\frac{\Delta}{2}\right)$, appeared in Eq. (11) are shown in Figs. 2(b) and (c). For $|\varepsilon| \leq \Delta / 2$, the two components are given respectively by

$$
R\left(\varepsilon+\frac{\Delta}{2}\right)+R\left(\varepsilon-\frac{\Delta}{2}\right)=1+\lambda, \quad R\left(\varepsilon+\frac{\Delta}{2}\right)-R\left(\varepsilon-\frac{\Delta}{2}\right)=-\frac{2(1-\lambda)}{\Delta} \varepsilon
$$

The constant and the proportionality factor in Eq. (12) are denoted by

$$
b_{\lambda} \equiv 1+\lambda, \quad k_{\lambda} \equiv-\frac{2(1-\lambda)}{\Delta}
$$

Then, $v_{c}$ is a probabilistic time-varying parameter which is independent of $\varepsilon$ and the state of the DLL is represented by three variables $\left(\varepsilon, v_{d}, v_{f}\right)$. Thus, Eq. (11) is linear with respect to $\left(\varepsilon, v_{d}, v_{f}\right)$ for $|\varepsilon|$ $\leq \Delta / 2$. However, term $v_{c} v_{d}$ does not let us apply superposition of respective responses of the DLL to MAI and channel noise. This makes analysis of the DLL difficult. The difficulty will be overcome by piecewise constant (PWC) approximation of the responses of the filters in the correlators to the MAI and the channel noise, as will be mentioned later in Section 4.1.

\section{Evolutional equation of probability distribution}

\subsection{Quantization of channel noise}

White Gaussian channel noise $\Gamma$ is quantized in order that the probability distribution of the state of the DLL can be described like that of a spatially-distributed probabilistic system. Quantized WGN can be expressed by the sum of binary i.i.d. sequences

$$
\begin{aligned}
\Gamma(t) \approx & \frac{\Gamma_{r m s}}{\sqrt{J_{N}}} \sum_{i=1}^{J_{N}} \gamma^{(i)}\left(t-t_{N}^{(i)}\right), \quad \gamma^{(i)}(t)=\sum_{n=-\infty}^{+\infty} \gamma_{n}^{(i)} \operatorname{rect}_{\Delta}(t-n \Delta), \\
& \gamma_{n}^{(i)} \in\{+1,-1\}, \quad \Gamma_{r m s}: \text { root mean square value of } \Gamma
\end{aligned}
$$

because i.i.d. sequences $\gamma^{(i)}$ have flat spectral density in the passband of the filters in the correlators and the binomial distribution approaches to Gaussian distribution at the limit $J_{N} \rightarrow \infty$.

In the rest of this section and the subsequent sections, the chip duration of spread-spectrum codes is normalized to $\Delta=1$. Let sample processes of MAI and the quantized channel noise in a time interval $[n, n+1]$ be denoted by $\mathcal{M A I}(n)$ and $\mathcal{N}(n)$. Since $J_{I}<L$ generally, the expected number of data signals $d^{(i)}$ changing in the interval is less than 1 . Hence, we may fix every data signal to $d^{(i)}$ $=1$ in the time interval. Then, the sample processes are designated by $c^{(i)}, \gamma^{(i)}$, and their switching time $n+t_{I}^{(i)}, n+t_{N}^{(i)}$, which is stated as follows:

$$
\begin{aligned}
\mathcal{M} \mathcal{A} \mathcal{I}(n) \equiv & \mathcal{M} \mathcal{A} \mathcal{I}\left(\boldsymbol{c}_{j_{I}}(n+1), \boldsymbol{c}_{i_{I}}(n), \boldsymbol{t}_{I}\right), \\
& \boldsymbol{c}_{j_{I}}(n+1)=\left(c^{(2)}(n+1), \cdots, c^{\left(J_{I}+1\right)}(n+1)\right), \quad j_{I}=\sum_{i=2}^{J_{I}+1}\left(\frac{c^{(i)}(n+1)+1}{2}\right) 2^{i-2}, \\
& \boldsymbol{c}_{i_{I}}(n)=\left(c^{(2)}(n), \cdots, c^{\left(J_{I}+1\right)}(n)\right), \quad i_{I}=\sum_{i=2}^{J_{I}+1}\left(\frac{c^{(i)}(n)+1}{2}\right) 2^{i-2}, \\
& \boldsymbol{t}_{I}=\left(t_{I}^{(2)}, \cdots, t_{I}^{\left(J_{I}+1\right)}\right), \quad 0<t_{I}^{(i)} \leq 1 \\
\mathcal{N}(n) \equiv & \left.\mathcal{N}\left(\gamma_{j_{N}}(n+1), \gamma_{i_{N}}(n), \boldsymbol{t}_{N}\right)\right), \\
& \gamma_{j_{N}}(n+1)=\left(\gamma^{(1)}(n+1), \cdots, \gamma^{\left(J_{N}\right)}(n+1)\right), \quad j_{N}=\sum_{i=1}^{J_{N}}\left(\frac{\gamma^{(i)}(n+1)+1}{2}\right) 2^{i-1}, \\
& \gamma_{i_{N}}(n)=\left(\gamma^{(1)}(n), \cdots, \gamma^{\left(J_{N}\right)}(n)\right), \quad i_{N}=\sum_{i=1}^{J_{N}}\left(\frac{\gamma^{(i)}(n)+1}{2}\right) 2^{i-1}, \\
& \boldsymbol{t}_{N}=\left(t_{N}^{(1)}, \cdots, t_{N}^{\left(J_{N}\right)}\right), \quad 0<t_{N}^{(i)} \leq 1
\end{aligned}
$$


Assume that the solution of Eq. (11) is analytically obtained when $c^{(1)}, \mathcal{M} \mathcal{A} \mathcal{I}(n), \mathcal{N}(n)$, and the DLL state $\left(\varepsilon(n), v_{d}(n), v_{f}(n)\right)$ at time $n$ are given. Then, the DLL state at time $n+1$ can be expressed with a vector function $\boldsymbol{f}$ as follows:

$$
\left(\varepsilon(n+1), v_{d}(n+1), v_{f}(n+1)\right)^{T}=\boldsymbol{f}\left(\left(\varepsilon(n), v_{d}(n), v_{f}(n)\right),\left(c^{(1)}(n+1), c^{(1)}(n)\right), \mathcal{M} \mathcal{A} \mathcal{I}(n), \mathcal{N}(n)\right)
$$

This is the difference equation governing the behavior of the DLL on discrete-time axis $n$.

\subsection{Probability distribution}

By the independence between MAI and channel noise, the independence between noise $\gamma^{(k)}$ and $\gamma^{(l)}$, and the Bayes' theorem, we have

$$
P\left(\boldsymbol{c}_{j_{I}}, \boldsymbol{\gamma}_{j_{N}} \mid \boldsymbol{c}_{i_{I}}, \boldsymbol{\gamma}_{i_{N}}\right)=P\left(\boldsymbol{\gamma}_{j_{N}}\right) P\left(\boldsymbol{c}_{j_{I}} \mid \boldsymbol{c}_{i_{I}}\right)
$$

Probability $P\left(\boldsymbol{\gamma}_{j_{N}}\right)$ and conditional probability $P\left(\boldsymbol{c}_{j_{I}} \mid \boldsymbol{c}_{i_{I}}\right)$ in Eq. (18) are given by

$$
\begin{aligned}
P\left(\gamma_{j_{N}}\right) & =\frac{1}{2^{J_{N}}} \\
P\left(\boldsymbol{c}_{j_{I}} \mid \boldsymbol{c}_{i_{I}}\right) & =\left(\frac{1-\lambda}{2}\right)^{\sum_{i=2}^{J_{I}+1}\left|\frac{c^{(i)}(n+1)-c^{(i)}(n)}{2}\right|}\left(\frac{1+\lambda}{2}\right)^{\sum_{i=2}^{J_{I}+1}\left|\frac{c^{(i)}(n+1)+c^{(i)}(n)}{2}\right|}
\end{aligned}
$$

Consider a $1 \Delta$-DLL receiving $c^{(1)}$, suffering from MAI $\sum_{i_{I}=2}^{J_{I}+1} c^{\left(i_{I}\right)}$ and quantized channel noise $\left(\Gamma_{r m s} / \sqrt{J_{N}}\right) \sum_{i_{N}=1}^{J_{N}} \gamma^{\left(i_{N}\right)}$ at time $n$, as shown in Fig. 3. Let a probability distribution of the state $s_{i_{I}, i_{N}} \equiv\left(\varepsilon, v_{d}, v_{f}\right)$ of a DLL given such conditions on the received signal values be denoted by

$$
\begin{aligned}
P\left(s_{i_{I}, i_{N}}(n)\right) \equiv & P\left(\left(\varepsilon(n), v_{d}(n), v_{f}(n)\right), c_{i_{I}}, \gamma_{i_{N}}\right), \\
& \leq i_{I}=\sum_{i=1}^{J_{I}+1} \frac{c^{(i)}(n)+1}{2} 2^{i-1} \leq 2^{J_{I}+1}-1, \\
& \leq i_{N}=\sum_{i=1}^{J_{N}} \frac{\gamma^{(i)}(n)+1}{2} 2^{i-1} \leq 2^{J_{N}}-1
\end{aligned}
$$

Notice that $\boldsymbol{c}_{i_{I}}$ in Eq. (21) includes $c^{(1)}$. The evolution of $P\left(\boldsymbol{s}_{i_{I}, i_{N}}\right)$ is described by the following equation:

$$
\begin{aligned}
P\left(\boldsymbol{s}_{j_{I}, j_{N}}(n+1)\right)= & \sum_{i_{I}=0}^{2^{J_{I}+1-1}} P\left(\boldsymbol{c}_{j_{I}}(n+1) \mid \boldsymbol{c}_{i_{I}}(n)\right) \sum_{i_{N}=0}^{2^{J_{N}}-1} \frac{1}{2^{J_{N}}} \\
& \times \int \delta\left(\boldsymbol{s}_{j_{I}, j_{N}}(n+1)^{T}-\boldsymbol{f}\left(\boldsymbol{s}_{i_{I}, i_{N}}(n),\left(c^{(1)}(n+1), c^{(1)}(n)\right), \mathcal{M} \mathcal{A} \mathcal{I}(n), \mathcal{N}(n)\right)\right) \\
& \times P\left(\boldsymbol{s}_{i_{I}, i_{N}}(n)\right) d \boldsymbol{s}_{i_{I}, i_{N}}(n)
\end{aligned}
$$

where we assumed that $\boldsymbol{t}_{I}$ and $\boldsymbol{t}_{N}$ are fixed.

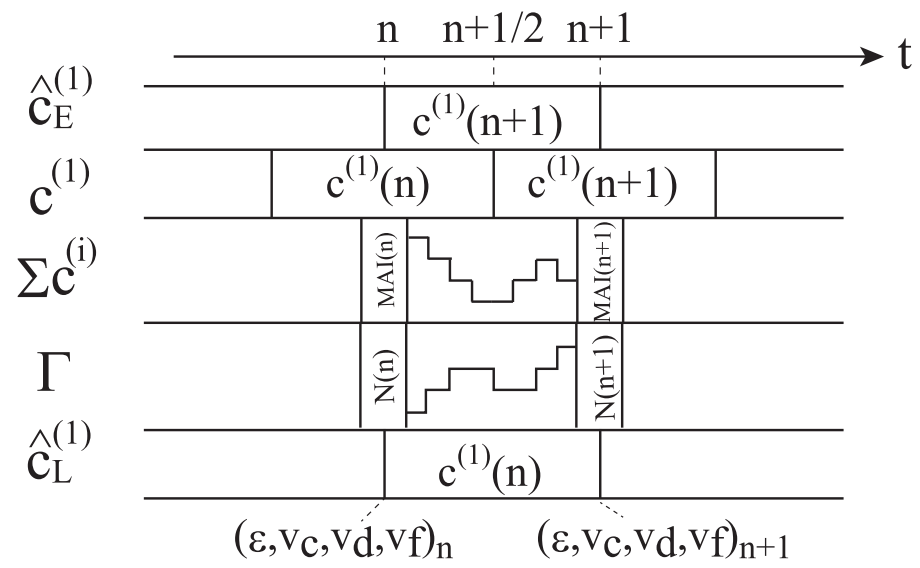

Fig. 3. Temporal relation among received sequence $c^{(1)}$, MAI signal, quantized channel noise, and the state of non-coherent $1 \Delta$-DLL. 


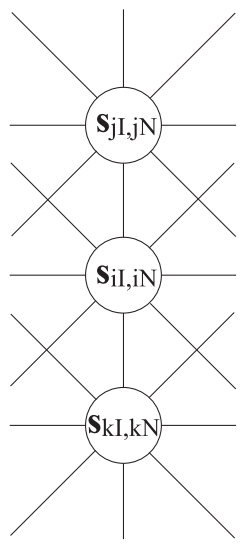

(a) Spatially-distributed infinite-range probabilistic system.

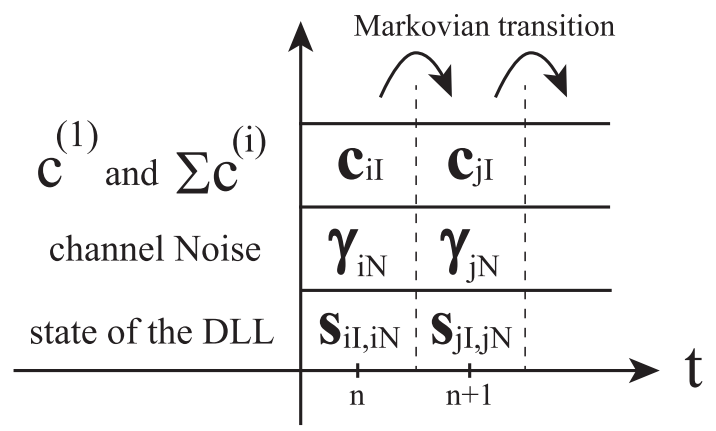

(b) Time variation of the state of the DLL.

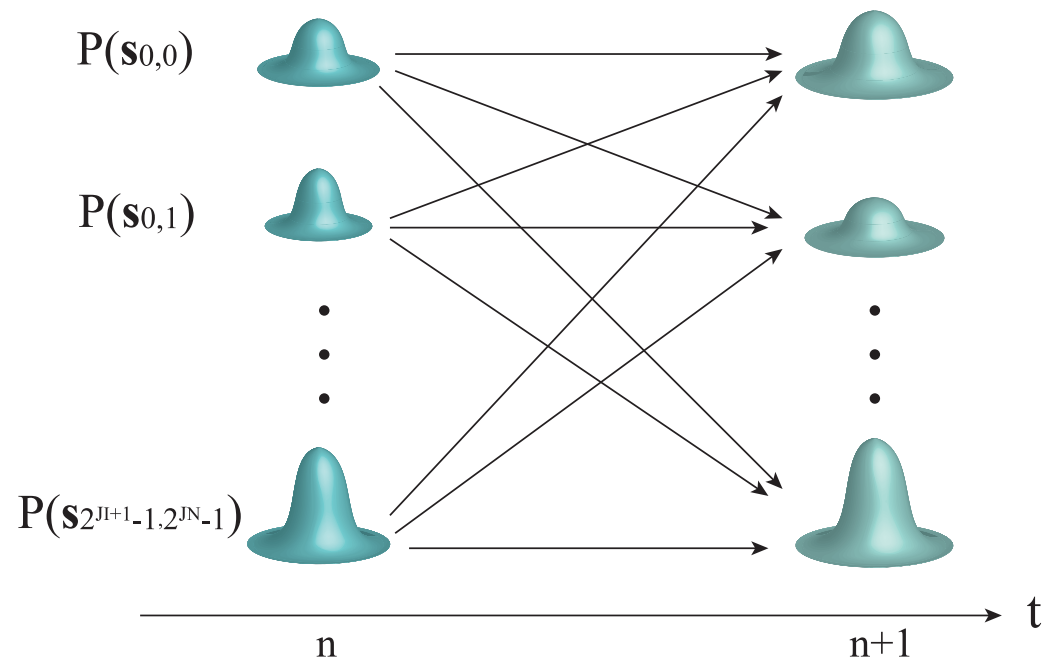

(c) Evolution of the probability distribution of the state of the DLL.

Fig. 4. Correspondance between the state of the DLL and the state of a spatially-distributed infinite-range probabilistic system.

\subsection{Comparison with spatially-distributed infinite-range probabilistic system}

Spatially-distributed infinite-range probabilistic systems consist of probabilistic elements each of which is coupled to all the other elements. We have shown that $P\left(s_{j_{I}, j_{N}}(n+1)\right)$ is determined by $P\left(s_{i_{I}, i_{N}}(n)\right)$ of all $\left(i_{I}, i_{N}\right)$, as given by Eq. (22). Thus, the evolution of the probability distribution of the state of the DLL corresponds to the evolution of that of a spatially-distributed infinite-range probabilistic system, that is a system consisting of all-coupled $\left(J_{I}+1\right) J_{N}$ elements whose probabilistic states are $s_{i_{I}, i_{N}}$. This correspondence is illustrated in Fig. 4 .

The MFA [15-17] estimates macroscopic characteristics of a spatially-distributed probabilistic system from the local interaction between the elements of the system with low computational complexity. Then, we consider that statistical characteristics of the DLL state are obtained with short computation time by a similar analysis to the MFA. In the next section, we will attempt to compute the moments of the state by an MFA-like analysis.

\section{Global moment computation}

\subsection{Piecewise constant approximation}

We denote common and differential outputs of the filters in the correlators in the absence of the MAI and the channel noise by $v_{c / d, o}$, the common and differential responses to the MAI by $v_{c / d, I, f r c}$, and the responses to the channel noise by $v_{c / d, N, f r c}$. Since $\left(\hat{c}_{E}^{(1)}+\hat{c}_{L}^{(1)}\right)\left(\hat{c}_{E}^{(1)}-\hat{c}_{L}^{(1)}\right)=0$, either $v_{c, I / N, f r c}$ or $v_{d, I / N, f r c}$ is the free response after the MAI and the channel noise are forced to zero. We denote the free responses by $v_{c / d, I / N, \text { free }}$. We decompose variables $v_{c}$ and $v_{d}$ into $v_{c / d, o}, v_{c / d, I, \text { frc } / \text { free }}$, and 
$v_{c / d, N, f r c / f r e e}$ and rewrite Eq. (11) as follows:

$$
\begin{aligned}
& \text { - If } \hat{c}_{E}^{(1)}+\hat{c}_{L}^{(1)}=0, \\
& \frac{d \varepsilon}{d t}=k_{o} v_{f} \\
& \text { - . - . . . . . . . } \\
& v_{c, o}=b_{\lambda} \\
& \frac{d v_{c, I, \text { free }}}{d t}=-\frac{1}{\tau_{p d}} v_{c, I, \text { free }} \\
& \frac{d v_{c, N, \text { free }}}{d t}=-\frac{1}{\tau_{p d}} v_{c, N, \text { free }} \\
& \frac{d v_{d, o}}{d t}=\frac{1}{\tau_{p d}}\left(k_{\lambda} \varepsilon-v_{d, o}\right) \\
& \frac{d v_{d, I, f r c}}{d t}=\frac{1}{\tau_{p d}}\left(\left(\hat{c}_{E}^{(1)}-\hat{c}_{L}^{(1)}\right) \sum_{i=2}^{J_{I}+1} c^{(i)}-v_{d, I, f r c}\right) \\
& \frac{d v_{d, N, f r c}}{d t}=\frac{1}{\tau_{p d}}\left(\left(\hat{c}_{E}^{(1)}-\hat{c}_{L}^{(1)}\right) \frac{\Gamma_{r m s}}{\sqrt{J_{N}}} \sum_{i=1}^{J_{N}} \gamma^{(i)}-v_{d, N, f r c}\right) \\
& \frac{d v_{f}}{d t}=\frac{1}{\tau_{f}}\left\{\left(v_{c, o}+v_{c, I, \text { free }}+v_{c, N, \text { free }}\right)\left(v_{d, o}+v_{d, I, f r c}+v_{d, N, \text { frc }}\right)-v_{f}\right\}
\end{aligned}
$$

- If $\hat{c}_{E}^{(1)}-\hat{c}_{L}^{(1)}=0$,

$$
\begin{aligned}
& \frac{d \varepsilon}{d t}=k_{o} v_{f} \\
& v_{c, o}=b_{\lambda} \\
& \frac{d v_{c, I, f r c}}{d t}=\frac{1}{\tau_{p d}}\left(\left(\hat{c}_{E}^{(1)}+\hat{c}_{L}^{(1)}\right) \sum_{i=2}^{J_{I}+1} c^{(i)}-v_{c, I, f r c}\right) \\
& \frac{d v_{c, N, f r c}}{d t}=\frac{1}{\tau_{p d}}\left(\left(\hat{c}_{E}^{(1)}+\hat{c}_{L}^{(1)}\right) \frac{\Gamma_{r m s}}{\sqrt{J_{N}}} \sum_{i=1}^{J_{N}} \gamma^{(i)}-v_{c, N, f r c}\right) \\
& \frac{d v_{d, o}}{d t}=\frac{1}{\tau_{p d}}\left(k_{\lambda} \varepsilon-v_{d, o}\right) \\
& \frac{d v_{d, I, \text { free }}}{d t}=-\frac{1}{\tau_{p d}} v_{d, I, \text { free }} \\
& \frac{d v_{d, N, \text { free }}}{d t}=-\frac{1}{\tau_{p d}} v_{d, N, \text { free }} \\
& -\cdots \ldots \ldots \ldots \\
& \frac{d v_{f}}{d t}=\frac{1}{\tau_{f}}\left\{\left(v_{c, o}+v_{c, I, f r c}+v_{c, N, f r c}\right)\left(v_{d, o}+v_{d, I, \text { free }}+v_{d, N, f r e e}\right)-v_{f}\right\}
\end{aligned}
$$

The three-equation groups in Eqs. (23) and (24) are obtained by applying the above mentioned variable decomposition to the second and the third equations in Eq. (11). As a result, LPF is described by the last equations of Eqs. (23) and (24).

If we neglect products $v_{c, I / N, f r c / f r e e} v_{d, o}$ and $v_{c, I / N, f r c / f r e e} v_{d, I / N, f r e e / f r c}$ and replace cascaded filters outputting $v_{d}$ and $v_{f}$ by one loop filter, the non-coherent DLL described by Eqs. (23) and (24) is equivalent to a linear model of the archetype DLL dealt with in [14]. For the linearized archetype DLL, the superposition principle is applicable to respective responses of the DLL to MAI and channel noise. The influences of the MAI and the channel noise are investigated respectively by the path integral analysis [14] and by deriving Fokker-Planck Equation [19]. In this paper, products $v_{c, I / N, f r c / f r e e} v_{d, o}$ and $v_{c, I / N, f r c / f r e e} v_{d, I / N, f r e e / f r c}$ remain in Eqs. (23) and (24) but $v_{c / d, I / N, f r c / f r e e}$ are PWC-approximated to overcome the difficulty mentioned in Section 2.2. 
Assume that the DLL state $\left(\varepsilon(n), v_{d}(n), v_{f}(n)\right)$ is given at time $n$ and that the switching time of $c^{(1)}$, $\mathcal{M A I}(n)$, and $\mathcal{N}(n)$ satisfy $t_{I}^{(1)}=0, t_{I}^{\left(i_{I}\right)} \leq t_{I}^{\left(i_{I}+1\right)}, t_{N}^{\left(i_{N}\right)} \leq t_{N}^{\left(i_{N}+1\right)}$. We approximate $v_{c / d, I / N, \text { frc } / \text { free }}(t)$ by PWC functions as follows:

$$
v_{c / d, I / N, f r c / \text { free }}(t) \approx v_{c / d, I / N, \text { frc } / \text { free }}\left(t_{I / N}^{(i+1)}\right), \quad t_{I / N}^{(i)}<t \leq t_{I / N}^{(i+1)}
$$

We then obtain analytical solution of Eqs. (23) and (24) in $\left(t_{I / N}^{(i)}, t_{I / N}^{(i+1)}\right]$. By connecting the solutions, the DLL state $\left(\varepsilon(n+1), v_{d}(n+1), v_{f}(n+1)\right)$ at time $n+1$ is attained. Then, Eq. (17) is approximated.

\subsection{Applying mean field analysis to the moment equation system}

The solution to a system of first-order differential equations

$$
\begin{aligned}
& \frac{d \boldsymbol{x}(t)}{d t}=\boldsymbol{M}(t) \boldsymbol{x}(t)+\boldsymbol{v}(t) \\
& \boldsymbol{x}(t) \in R^{N}, \quad \boldsymbol{M}(t): N \times N \text { time-varying matrix, } \quad \boldsymbol{v}(t) \in R^{N}: \text { time varying vector }
\end{aligned}
$$

with initial state $\boldsymbol{x}_{0}$ is represented by using the solution $\boldsymbol{x}(t)=\boldsymbol{R}(t, 0) \boldsymbol{x}_{0}$ to $d \boldsymbol{x} / d t=\boldsymbol{M} \boldsymbol{x}$ as

$$
\boldsymbol{x}(t)=\boldsymbol{R}(t, 0) \boldsymbol{x}_{0}+\int_{0}^{t} \boldsymbol{R}\left(t, t^{\prime}\right) \boldsymbol{v}\left(t^{\prime}\right) d t^{\prime}, \quad \boldsymbol{R}\left(t, t^{\prime}\right): N \times N \text { time-varying matrix }
$$

Then, the difference equation which is approximate to Eq. (17) by the procedure in Section 4.1 is expressed as

$$
\begin{aligned}
& \boldsymbol{s}_{j_{I}, j_{N}}(n+1)=\boldsymbol{A}_{j_{I}, j_{N}, i_{I}, i_{N}} \boldsymbol{s}_{i_{I}, i_{N}}(n)+\boldsymbol{B}_{j_{I}, j_{N}, i_{I}, i_{N}}, \\
& \boldsymbol{A}_{j_{I}, j_{N}, i_{I}, i_{N}}: 3 \times 3 \text { matrix, } \quad \boldsymbol{B}_{j_{I}, j_{N}, i_{I}, i_{N}}: 3 \text {-dimensional vector }
\end{aligned}
$$

From Eqs. (22) and (28), the following difference equation of the expectation of $\boldsymbol{s}_{i_{I}, i_{N}}$ is obtained:

$$
\begin{aligned}
& <s_{j_{I}, j_{N}}(n+1)>=\sum_{i_{I}=0}^{2^{J_{I}+1}-1} P\left(\boldsymbol{c}_{j_{I}} \mid \boldsymbol{c}_{i_{I}}\right) \sum_{i_{N}=0}^{2^{J_{N}}-1} \frac{1}{2^{J_{N}}} \boldsymbol{A}_{j_{I}, j_{N}, i_{I}, i_{N}}<\boldsymbol{s}_{i_{I}, i_{N}}(n)> \\
& +\sum_{i_{I}=0}^{2^{J_{I}+1}-1} P\left(\boldsymbol{c}_{j_{I}} \mid \boldsymbol{c}_{i_{I}}\right) \sum_{i_{N}=0}^{2^{J_{N}}-1} \frac{1}{2^{J_{N}}} \boldsymbol{B}_{j_{I}, j_{N}, i_{I}, i_{N}}
\end{aligned}
$$

We can substantiate numerically that $\boldsymbol{A}_{j_{I}, j_{N}, i_{I}, i_{N}}$ is almost independent of $\left(j_{I}, j_{N}, i_{I}, i_{N}\right)$, that is received signal $c^{(1)}$, MAI, and the quantized noise. This is because $\boldsymbol{x}_{0}$ and $\boldsymbol{v}(t)$ are separated in Eq. (27) and products $v_{d, o} v_{c, I, f r e e / f r c}, v_{d, o} v_{c, N, f r e e / f r c}$ in Eqs. (23) and (24) are small. Then, we replace all the transition matrices $\boldsymbol{A}_{j_{I}, j_{N}, i_{I}, i_{N}}$ by a constant matrix $\boldsymbol{A}_{1}$,

$$
\boldsymbol{A}_{j_{I}, j_{N}, i_{I}, i_{N}}=\boldsymbol{A}_{1} \text { for all } j_{I}, j_{N}, i_{I}, i_{N}
$$

Let global average of the DLL state be defined as

$$
<\boldsymbol{s}(n)>=\frac{1}{2^{J_{I}+1} 2^{J_{N}}} \sum_{i_{I}=0}^{2^{J_{I}+1}-1} \sum_{i_{N}=0}^{2^{J_{N}}-1}<\boldsymbol{s}_{i_{I}, i_{N}}(n)>
$$

From Eq. (30) and that

$$
\sum_{i_{I}=0}^{2^{J_{I}+1}-1}\left(P\left(\boldsymbol{c}_{j_{I}} \mid \boldsymbol{c}_{i_{I}}\right)-\frac{1}{2^{J_{I}+1}}\right)=0, \quad \sum_{j_{I}=0}^{2^{J_{I}+1}-1}\left(P\left(\boldsymbol{c}_{j_{I}} \mid \boldsymbol{c}_{i_{I}}\right)-\frac{1}{2^{J_{I}+1}}\right)=0
$$

we obtain

$$
<\boldsymbol{s}(n+1)>=\boldsymbol{A}_{1}<\boldsymbol{s}(n)>+\frac{1}{2^{J_{I}}+12^{J_{N}}} \sum_{j_{I}=0}^{2^{J_{I}+1}-1} \sum_{j_{N}=0}^{2^{J_{N}}-1} \sum_{i_{I}=0}^{2^{J_{I}+1}-1} P\left(\boldsymbol{c}_{j_{I}} \mid \boldsymbol{c}_{i_{I}}\right) \sum_{i_{N}=0}^{2^{J_{N}}-1} \frac{1}{2^{J_{N}}} \boldsymbol{B}_{j_{I}, j_{N}, i_{I}, i_{N}}
$$


As the MFA derives the macroscopic states of a spatially-distributed probabilistic system, the above procedure leads to difference Eq. (33) of the averaged DLL state.

By the similar manner to the above procedure, difference equations of the 2 nd and the higher-order moments of the state variables $\varepsilon, v_{d}$, and $v_{f}$ are derived. Computing steady solutions of the difference equations, we obtain averages, variances, and the higher-order moments of the stationary state of the DLL. In addition to the stationary state, the duration of the transition from an initial state to the stationary state is estimated by linearizing the difference equations around the steady solutions.

\section{Estimation of phase tracking error}

\subsection{Stationary moment analysis}

We will compute the variance $\left\langle\varepsilon^{2}>\right.$ of the phase tracking error of a non-coherent $1 \Delta$-DLL used in asynchronous DS-CDMA communication with i.i.d. spreading sequences of eigenvalue $\lambda=0$ and Markovian spreading sequences of $\lambda=-2+\sqrt{3}$. The latter sequences employed in asynchronous DS-CDMA are reported to minimize the variance of the cross-correlation between the sequences $[9$, 10].

The parameters of the DLL to be analyzed are as follows: Time constant $\tau_{p d}$ of the filters in the two correlators is $10 \Delta$, which satisfies the requirement $1 \Delta<\tau_{p d}$ in Section 2. VCO gain is $k_{o}=2 \times 10^{-3}$. Time constant $\tau_{f}$ of LPF is $10 \Delta$. These two parameters $k_{o}$ and $\tau_{f}$ are decided from the view points of the frequency precision of practical VCO, the sensitivity of the DLL to MAI and channel noise, and the dynamical tracking performance [14]. As we derived Eq. (33) for the average of the DLL state, the difference equation of the variances of the state is obtained by the MFA-like procedure in Section 4 in the following form:

$$
\begin{aligned}
\boldsymbol{m}_{2}(n+1)= & \boldsymbol{A}_{2} \boldsymbol{m}_{2}(n)+\boldsymbol{B}_{2}\left(<\varepsilon(n)>,<v_{d}(n)>,<v_{f}(n)>\right), \\
& \boldsymbol{m}_{2}(n) \equiv\left(<\varepsilon(n)^{2}>,<\varepsilon(n) v_{d}(n)>, \cdots,<v_{f}(n)^{2}>\right)^{T}, \\
& \boldsymbol{A}_{2}: 6 \times 6 \text { matrix, } \quad \boldsymbol{B}_{2}: \text { 6-dimensional vector }
\end{aligned}
$$

The elements of vector $\boldsymbol{B}_{2}$ contain the state average $\langle\varepsilon(n)\rangle,\left\langle v_{d}(n)\right\rangle$, and $\left\langle v_{f}(n)\right\rangle$.

Figure 5 shows variance $\left\langle\varepsilon^{2}>\right.$ versus user number $J_{I}+1$ curves obtained from Eq. (34) and by numerical simulation of the DLL under different noise power, $\Gamma_{r m s}=0,1 / \sqrt{2}$, and 1 . In the moment estimation according to Section 4 , it is naturally postulated that the spreading sequences $c^{(i)}(t)$ are infinite in length. Then, the numerical simulation was also carried out with aperiodic sequences. The postulation is permissible since the necessity of phase tracking increases with the length of spreading codes. However, we may not be careful about the length because the phase tracking performance is almost independent of the length. When the numbers $J_{I}$ and $J_{N}$ of MAI and quantized channel noise components $c^{\left(i_{I}\right)}$ and $\gamma^{\left(i_{N}\right)}$ are large, the moment computation requires large storage. In this case, we decreased the number of the DLL states so that any two DLL states $\boldsymbol{s}_{i_{I}, i_{N}}$ and $\boldsymbol{s}_{j_{I}, j_{N}}$ did not have the same second suffixes, that is $i_{N} \neq j_{N}$. The time spent for computing the variance by the method in Section 4 was about 1/1000 compared to that by the path integral method [14] when no channel noise was added to the DLL.

Figure 5 indicates that replacing i.i.d. spreading sequences with Markovian sequences of $\lambda=-2+\sqrt{3}$ reduces the phase tracking error. This result is explained qualitatively as follows: For a linearized DLL [1-5], the influence of MAI and channel noise to the phase tracking error is expressed by the following lowpass transfer function in the frequency domain:

$$
\frac{\varepsilon}{\mathcal{M A \mathcal { I }}+\mathcal{N}}(\omega)=\frac{b_{\lambda} k_{o}}{j \omega\left(j \omega+\frac{1}{\tau_{p d}}\right)\left(j \omega+\frac{1}{\tau_{f}}\right)-b_{\lambda} k_{o} k_{\lambda}}
$$

Constant $b_{\lambda}$ and proportionality factor $k_{\lambda}$ given by Eq. (13) depend on eigenvalue $\lambda$. As $\lambda$ decreases from zero, $b_{\lambda}$ decreases and $k_{\lambda}$ increases. Then, the gain and the bandwidth of the transfer function decrease as $\lambda$ decreases to negative. This means that to employ the Markovian sequences as spreading codes reduces the influence of both MAI and channel noise to the phase tracking. Even if MAI does not exist, the Markovian sequences are useful. 


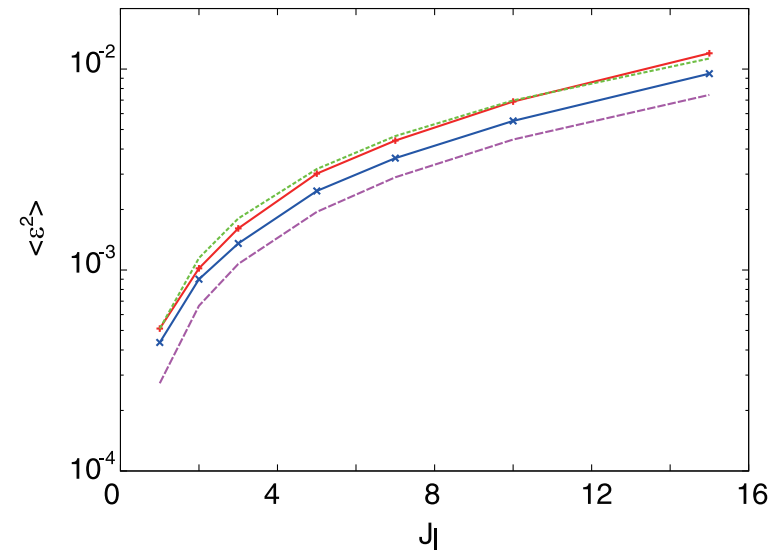

(a) $\Gamma_{r m s}=0$.

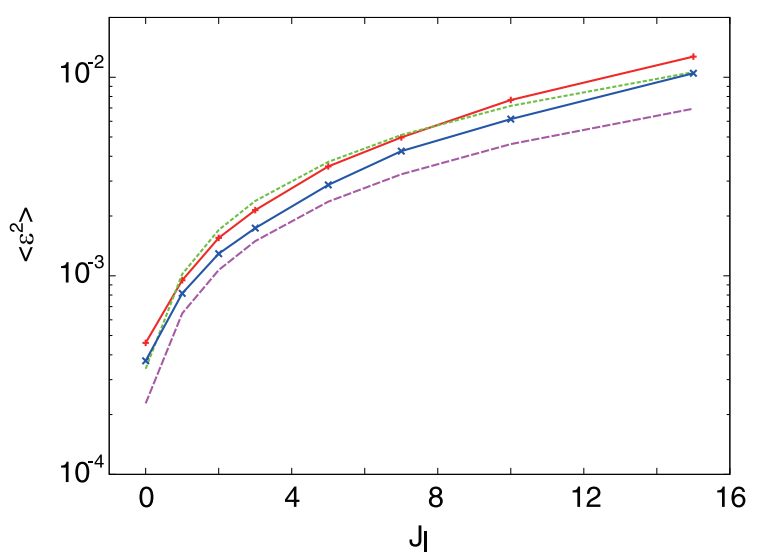

(c) $\Gamma_{r m s}=1$.

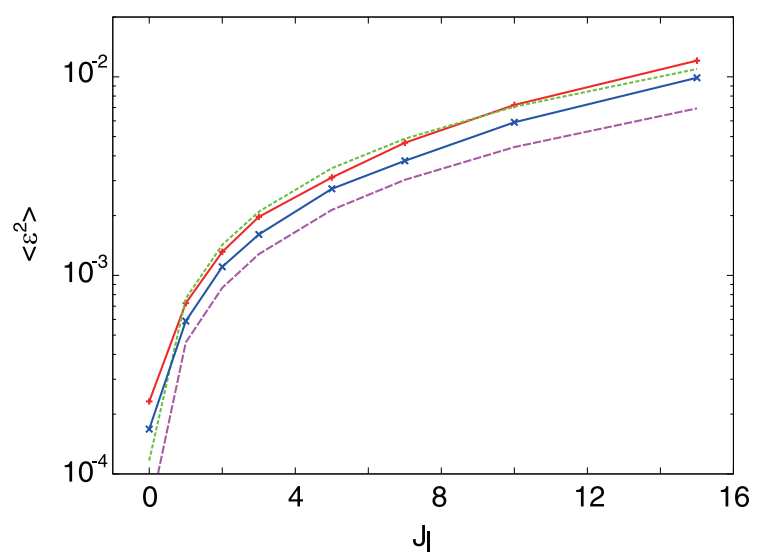

(b) $\Gamma_{r m s}=1 / \sqrt{2}$.

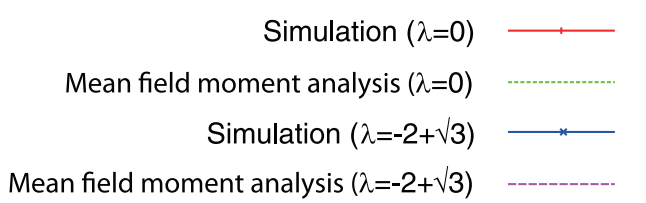

Fig. 5. Stationary phase tracking error versus the number of users. The spreading sequences are i.i.d. sequences of eigenvalue $\lambda=0$ and Markovian sequences of $\lambda=-2+\sqrt{3}$.

Maximum-length sequences (M-sequences) [1,2] have long been used in spread spectrum communications. Roughly speaking, long M-sequences possess the same correlation property and spectral distribution with those of i.i.d. sequences [9]. Therefore, $1 \Delta$-DLLs used in the CDMA communications with long M-sequences are considered to have almost the same phase tracking error performance with those shown in Fig. 5 for i.i.d. sequences.

Gold codes are prevalent in chip-synchronous CDMA communications because of their distinguished cross-correlation property $[1,2]$. It is interesting to investigate phase tracking error of $1 \Delta$-DLLs when Gold codes are applied to asynchronous CDMA communications. Figure 6 shows the variance of the phase tracking error along with the error variances when Markovian and i.i.d. sequences are employed. They are obtained by numerical simulation with the codes of length $2^{11}-1$. Note that the phase tracking performance is almost independent of the code length, as we mentioned above. The variance is larger when Gold codes are employed than when Markovian sequences are used. In addition, we can not necessarily say that Gold codes are more effective than i.i.d. sequences to decrease the error in asynchronous use.

\subsection{Transient time analysis}

We combine Eqs. (33) and (34) into one equation and linearize it. We express the linearized equation as

$$
\begin{aligned}
& \boldsymbol{m}_{1,2}(n+1)=\boldsymbol{A}_{1,2} \boldsymbol{m}_{1,2}(n)+\boldsymbol{B}_{1,2}, \\
& \quad \boldsymbol{m}_{1,2}(n) \equiv\left(<\varepsilon(n)>,<v_{d}(n)>,<v_{f}(n)>,<\varepsilon(n)^{2}>,<\varepsilon(n) v_{d}(n)>, \cdots,<v_{f}(n)^{2}>\right)^{T},
\end{aligned}
$$

$\boldsymbol{A}_{1,2}: 9 \times 9$ matrix, $\quad \boldsymbol{B}_{1,2}: 9$-dimensional vector 


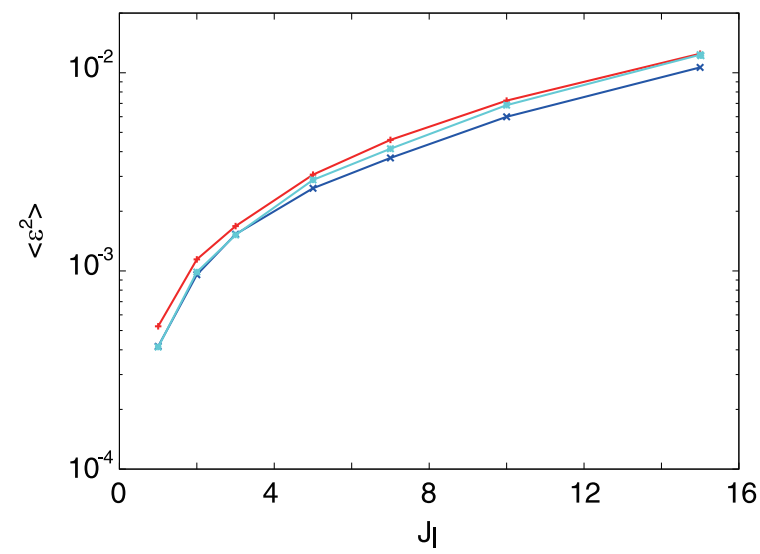

(a) $\Gamma_{r m s}=0$.

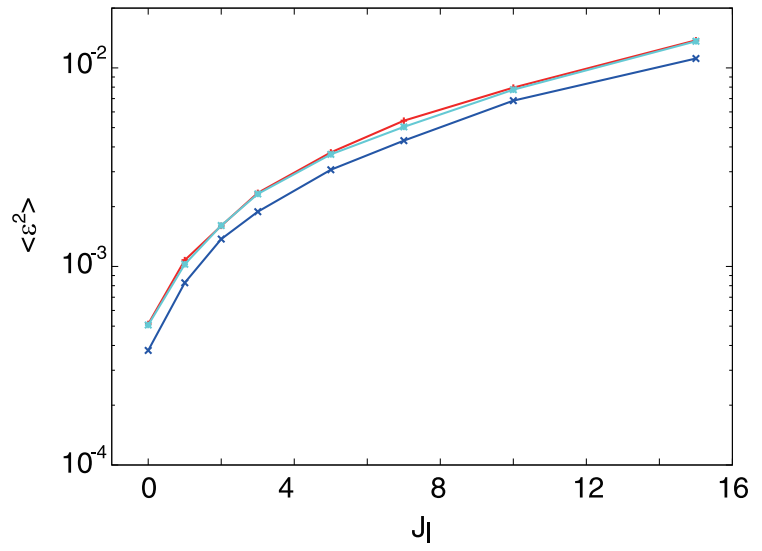

(b) $\Gamma_{r m s}=1$.

Simulation $(\lambda=0) \longrightarrow \quad$ Simulation $(\lambda=-2+\sqrt{3}) \longrightarrow \times \quad$ Simulation (Gold codes)

Fig. 6. Stationary phase tracking error versus the number of users. The spreading sequences are Gold codes, i.i.d. sequences $(\lambda=0)$, and Markovian sequences $(\lambda=-2+\sqrt{3})$. Their length is $2^{11}-1$.

We parameterize the maximum eigenvalue of $\boldsymbol{A}_{1,2}$ with $\lambda$ and denote it by $\Lambda(\lambda)$. Let $T_{10}(\lambda)$ be the time satisfying $\varepsilon\left(T_{10}(\lambda)\right)^{2}=(\varepsilon(0) / 10)^{2}$ when the DLL receives signals spread by sequences of eigenvalue $\lambda$. Since the following equality is expected

$$
\Lambda(\lambda)^{T_{10}(\lambda)}=\Lambda(0)^{T_{10}(0)}
$$

the transition time ratio $T_{10}(\lambda) / T_{10}(0)$ may be estimated by

$$
R(\lambda)=\frac{\ln \Lambda(0)}{\ln \Lambda(\lambda)}
$$

The time ratio is considered to be $T_{10}(-2+\sqrt{3}) / T_{10}(0)>1$ since the bandwidth of the transfer function (35) becomes narrower as $\lambda$ decreases from zero to negative. By simulating numerically the DLL with initial value $\varepsilon(0)=0.4 \Delta$, we evaluated the ratio as $T_{10}(-2+\sqrt{3}) / T_{10}(0)=1.083$. By computing the maximum eigenvalue of $\boldsymbol{A}_{1,2}$, we obtained $R(-2+\sqrt{3})=1.085$. Difference equations of the moments of the DLL state are useful to estimate the dependency of the dynamical response time of the DLL on eigenvalue $\lambda$.

\section{Conclusions}

We have presented an analysis of probabilistic state of a non-coherent $1 \Delta$-DLL in DS-CDMA communication employing Markovian spreading sequences. In this analysis, the following two approximations are introduced: First, additive white Gaussian channel noise is approximated as a sum of binary i.i.d. sequences. By this quantization of the noise, the evolution of the probability distribution of the state of DLL is represented like the evolution of that of a spatially-distributed infinite-range probabilistic system. Secondly, outputs of the filters in the correlators of the DLL are PWC-approximated. By this approximation, the behavior of the DLL can be described by a system of discrete-time first-order difference equations with linear terms independent of received signal, MAI, and the quantized noise. Then, in computing the moments of the DLL state, we can apply a similar analysis to the MFA which is used to estimate macroscopic characteristics of a spatially-distributed probabilistic system from the local interaction between the elements of the system with low computational complexity. The low complexity makes it possible to analyze the behavior of not only second-order archetype DLLs but also higher-order non-coherent DLLs in the presence of both MAI and channel noise. It is found from the presented analysis that replacing i.i.d. spreading sequences with Markovian spreading sequences of eigenvalue $\lambda=-2+\sqrt{3}$ reduces the phase tracking error of the DLL. The time spent for estimating the error by the MFA-like method is about 1/1000 compared with that by the path integral method. 


\section{References}

[1] K.S. Zigangirov, Theory of Code Division Multiple Access Communication, IEEE Press, 2004.

[2] M.A. Abu-Rgheff, Introduction to CDMA Wireless Communications, Academic Press, 2007.

[3] J. Caffery, Jr. and G.L. Stuber, "Effects of multiple-access interference on the noncoherent delay lock loop," IEEE Trans. Commun., vol. 48, no. 12, pp. 2109-2119, December 2000.

[4] S.E. Hong, S.Y. Yoon, H.S. Lee, and J. Ahn, "Performance analysis of non-coherent delay-locked loop in multiple access interference," IEICE Trans. Commun., vol. E78-B, no. 6, pp. 935-941, June 1995.

[5] M. Latva-aho and J. Lilleverg, "Delay trackers for multiuser CDMA receivers," Proc. IEEE Int'l Conf. on Universal Personal Commun., pp. 326-330, August 1996.

[6] G. Mazzini, G. Setti, and R. Rovatti, "Chaotic complex spreading sequences for asynchronous DS-CDMA - Part I," IEEE Trans. Circuit Syst. I, vol. 44, no. 10, pp. 937-947, October 1997.

[7] R. Rovatti and G. Mazzini, "Interference in DS-CDMA systems with exponentially vanishing autocorrelations: Chaos-based spreading is optimal," Electron. Lett., vol. 34, no. 20, pp. 19911993, October 1998.

[8] G. Mazzini, R. Rovatti, and G. Setti, "Interference minimization by autocorrelation shaping in asynchronous DS-CDMA systems: Chaos-based spreading is nearly optimal," Electron. Lett., vol. 35, no. 13, pp. 1054-1055, June 1999.

[9] T. Kohda, "Information sources using chaotic dynamics," Proceedings of the IEEE, vol. 90, no. 5, pp. 641-661, May 2002.

[10] T. Kohda and H. Fujisaki, "Variance of multiple access interference code average agains data average," Electron. Lett., vol. 38, no. 20, pp. 1717-1719, September 2000.

[11] T. Kohda, Y. Matsumura, and Y. Jitsumatsu, "Bit-error rate in an asynchronous DS/CDMA system using Markovian SS codes," IEEE Int'l Symp. on Spread-Spectrum Tech. Appli., pp. 571575, September 2002.

[12] R. Rovatti, G. Mazzini, and G. Setti, "On the ultimate limits of chaos-based asynchronous DSCDMA-I: Basis definitions and results," IEEE Trans. Circuit Syst. I, vol. 51, no. 7, pp. 13361347, July 2004.

[13] R. Rovatti, G. Mazzini, and G. Setti, "On the ultimate limits of chaos-based asynchronous DS-CDMA-II: Analytical results and asymptotics," IEEE Trans. Circuit Syst. I, vol. 51, no. 7, pp. 1348-1364, July 2004.

[14] K. Nagata, H. Fujisaka, T. Kamio, C. Ahn, and K. Haeiwa, "Steady-state analysis of delaylocked loops tracking binary Markovian sequences," NOLTA, vol. 1, no. 1, pp. 153-165, October 2010.

[15] D.A. Lavis and G.M. Bell, Statistical Mechanics of Lattice Systems 1: Closed-Form and Exact Solutions, Springer-Verlag, 1999.

[16] M. Opper and D. Saad (eds), Advanced Mean Field Methods - Theory and Practice -, MIT Press, 2001.

[17] H. Nishimori, Statistical Physics of Spin Glasses and Information Processing: An Introduction, Oxford University Press, 2001.

[18] T. Tanaka, "A statistical-mechanics approach to large-system analysis of CDMA multiuser detectors," IEEE Trans. Information Theory, vol. 48, no. 11, pp. 2888-2910, November 2002.

[19] H. Risken, The Fokker-Plank Equation (2nd ed.), Springer-Verlag, 1989. 